\title{
Hyperspectral imaging applied to end-of-life (EOL) concrete recycling
}

\author{
Giuseppe Bonifazi, Roberta Palmieri and Silvia Serranti \\ Sapienza - Universitá di Roma \\ Dipartimento di Ingegneria Chimica Materiali Ambiente \\ Via Eudossiana, 1800184 Rome, Italy
}

\begin{abstract}
The recovery of materials from Demolition Waste (DW) is a significant target of the recycling industry and it is important to know which materials are present in order to set up efficient sorting and/or quality control actions. The implementation of an automatic recognition system of recovered products from EndOf-Life (EOL) concrete materials can be an useful way to maximize DW conversion into secondary raw materials. In this paper a new approach, based on HyperSpectral Imaging (HSI) sensors, is investigated in order to develop suitable and low cost strategies finalized to the preliminary detection and characterization of materials constituting DW flow stream. The described HSI quality control approach is based on the utilization of a device working in the near infrared range (1000-1700 nm). Acquired hyperspectral images were analyzed. Different chemometric methods were applied. Results showed that it is possible to recognize DW materials and to distinguish the recycled aggregates from the investigated contaminants (brick, gypsum, plastic, wood and foam).
\end{abstract}

\section{Introduction}

The huge amount of generated Construction and Demolition Waste (CDW) constitutes a serious problem. Eurostat estimated a total production of 970 million tons/year, representing an average value of almost 2.0 ton/ per capita only in Europe. This estimation is lower than the reality because this type of waste is often illegally dumped [1]. On the other hand, during the last century, the use of global materials increased eight-fold with almost 60 billion tons currently used per year. 
It has been forecast that the demand for these materials will double by 2050. CDW recycling is of fundamental importance because it allows to have "new materials" to use but also because it reduces environmental pressure, preventing the increase of land use for waste disposal and also avoiding the exploitation of non-renewable raw materials. Environmental impacts linked with the non-renewable raw materials extraction include extensive deforestation, soil loss, air and water reserves pollution. In this context, the benefits of effective CDW recycling are evident. The construction sector addressed significant efforts to find methods to re-use the high volumes of CDW generated each year. A practical solution can be to produce recycled aggregates from End-OfLife (EOL) concrete as a replacement for natural aggregates. In this way, adopting recycling strategies to the EOL concrete, the improvement of environment condition and the secondary raw materials collection can be achieved at the same time [2]. In order to obtain marketable recycled aggregates , pollutants, such as brick, plastic, wood, gypsum, etc., usually present into a Demolition Waste (DW) stream, must be absent or under the limits required by the market. Therefore, the realization of an automatic system able to identify DW materials is desirable in order to make easier their conversion into useful secondary raw materials. Therefore the possibility to develop efficient, reliable and low cost sensing technologies able to perform detection/control actions during all recycling steps has to be explored. 
Main aim of this study was the DW stream classification by optical sensors in order to identify concrete aggregates and unwanted contaminants (i.e. brick, wood, plastic, gypsum and foam). The developed classification method is based on the utilization of HyperSpectral Imaging (HSI) sensing devices acting in the NIR range (1000-1700 nm). An integrated hardware and software architecture constitutes the HSI system: it allows to digitally capture and handle spectra, as they result along a pre-defined alignment on a surface sample properly energized [3], [4]. Hyperspectral sensor produces a 3D dataset, the "hypercube", characterized by two spatial and one spectral dimensions. In the hyperspectral images, the wavelength bands are typically in an equally spaced sequence, so a full spectrum is obtained for each pixel that can be analyzed giving spectral information about samples [5]. Samples spectral behavior is linked to their physical-chemical features according to the different investigated wavelengths and it can be used for a rapid materials recognition/classification.

\section{Materials and methods}

\subsection{Samples}

Strukton company (NL) provided samples representative of some contaminants of concrete collected after the demolition of a building in Groningen (NL). More in detail, samples are particles of wood, plastic, gypsum, brick and foam. The investigated concrete aggregates came from the same demolition waste, but after Advanced Dry Recovery (ADR) processing in TUDelft (Delft, NL) [6]. This study was thus carried in order to define the best classification strategy for identification of aggregates and pollutant materials. In order to reach this goal, HSI model classification was performed using two experimental set-up, as described in the following.

Experimental set-up 1: the training sample set. Particles of brick (2 particles), aggregates (3 particles), wood (1 particle), gypsum (3 particles), foam ( 3 particles) and plastic (4 particles), arranged in 7 lines, were acquired generating a training image sample set used to build the classification model (Figure 12.1) [7].

Experimental set-up 2: validation sample set. Twelve random particles were arranged in three separated parallel lines, obtaining 4 different material 


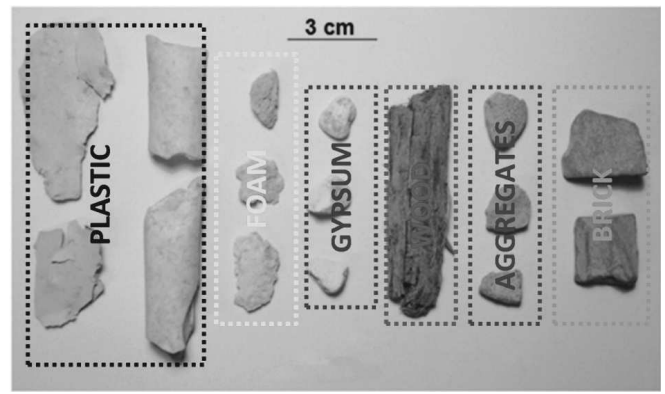

Figure 12.1: Digital image representative of the training sample set (Experimental set up 1).

particles for each row and a validation sample set was thus acquired (Figure 12.2). This second acquisition was carried out in order to validate the classification model developed through the training sample set.

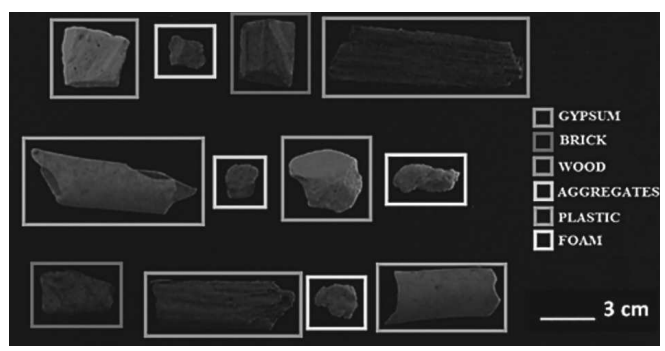

Figure 12.2: Digital image representative of the validation sample set (Experimental set up 2). Each material, belonging to the same class, is identified by a frame of the same color.

\subsection{The hyperspectral imaging (HSI) system}

Hyperspectral images were acquired adopting a specifically designed platform by DV srl (Padova, Italy) located at the Laboratory for Particles and Particulate Solids Characterization (Latina, Italy) of the Department of Chemical Materials and Environment Engineering (Sapienza - 
University of Rome, Rome, Italy). The adopted platform is able to perform both static and dynamic analysis, indeed it is equipped with a conveyor belt (width $=26 \mathrm{~cm}$ and length $=160 \mathrm{~cm}$ ) with adjustable speed to simulate the behavior of material flow-stream in a real industrial plant. Moreover the system consists of a NIR Spectral Camera ${ }^{\mathrm{TM}}$ (Specim, Finland) equipped with an ImSpector N17E ${ }^{\mathrm{TM}}$ imaging spectrograph working with a spectral sampling/pixel of $2.6 \mathrm{~nm}$, coupled with a Te-cooled InGaAs photodiode array sensor. The device works as a push-broom type line scan camera allowing the acquisition of spectral information for each pixel in the line. In order to provide the required energy for the process, a diffuse light cylinder containing five halogen lamps producing a continuous spectrum signal optimized for spectra acquisition in the NIR wavelength range $(1000-1700 \mathrm{~nm})$ is present.

A PC unit equipped with a specifically developed software (Spectral Scanner $\left.{ }^{\mathrm{TM}} \mathrm{v} .2 .3\right)$, allowing to acquire, collect and manage the spectra, controls the HSI system. Hyperspectral data were acquired in the 880-1720 nm wavelength range, with a spectral resolution of $7 \mathrm{~nm}$, for a total of 121 wavelengths. The width of the images was 320 pixels and the number of frames was variable according to the length of the image to acquire. A calibration, obtained recording two reference images (one for black and one for white) was carried out preliminarily. Black image (B) was acquired in order to remove the dark current effect of the camera sensor covering the camera lens with its cap. The white image $(\mathbf{W})$ was acquired adopting a standard white ceramic tile under the same condition of the raw image. Corrected hyperspectral image (I) was obtained applying the following equation to the original sample image $\mathbf{S}$ :

$\mathbf{I}=\frac{\mathbf{S}-\mathbf{B}}{\mathbf{W}-\mathbf{B}} \cdot 100$

\section{Spectral data analysis}

The PLS_Toolbox (Version 7.8, Eigenvector Research, Inc., Wenarchee, USA) running under Matlab®(Version 7.5, The Mathworks, Inc., Natick, Massachusetts, USA) environment was used in order to analyze hyperspectral images. After a preliminary pre-processing procedure, chemometric techniques were applied. Principal Components Analysis 
(PCA) was used in order to explore the data, to define classes and to evaluate the best algorithms for successive classification models, while for classification purpose Partial Least-Squares Discriminant Analysis (PLS-DA) was adopted.

\subsection{Spectra pre-processing and exploratory analysis}

In order to remove effects due to the background noise, at the beginning and at the end of the acquisition range, the raw spectra were cut: spectral variables were thus reduced from 121 to 93 obtaining a new investigated wavelength interval (1006 to $1650 \mathrm{~nm}$ ).

To highlight samples spectral differences and to reduce the impact of possible external sources of variability, hyperspectral data were preprocessed adopting a combination of three algorithms was applied: Mean Centering (MC), Detrend and Standard Normal Variate (SNV). MC is one of the most common pre-processing methods calculating the mean of each column and subtracting this from the column: each row of the mean-centered data includes only how that row differs from the average sample in the original data matrix. Detrend algorithm removes constant, linear, or curved offset; while SNV is a weighted normalization adopted to solve scaling or gain effects due to path length effects, scattering effects (depending on the physical nature of the material and the particle size), source or detector variations, or other general instrumental sensitivity effects [8]. After preprocessing, Principal Component Analysis (PCA) was applied as exploratory data analysis [9]. PCA projects the samples into a low dimensional subspace, whose axes (the principal components, PCs) point in the direction of maximal variance, compressing the data. Looking at the distribution of samples into the PCs space, it is possible to interpret differences and similarities among them: the more they are grouped, the more they have similar spectral features. Furthermore, PCA highlights the presence of trends among samples, giving preliminary information about distribution of different specimen on an image [10].

\subsection{Partial Least-Square Discriminant Analysis}

Partial Least-Square Discriminant Analysis (PLSDA) was adopted in order to build a predictive model able to classify aggregates and pollu- 
tants. This technique is a supervised classification method as requires a prior data knowledge [11] and it is a linear method based on the use of partial least squares regression. Starting from samples with known classes, a discriminant function is built to be used to classify new unknown samples, made of the same material of the known ones. PLS-DA builds a subspace of latent variables (LVs), which are liner combinations of the original variables, and it looks for the LVs with a maximum covariance. It is necessary to evaluate the optimal dimension of the LVs subspace in order to perform the best classification model as possible [12]. The PLS-DA model allows to assign to each unknown sample (in this case, pixel) only one of the available defined classes, according to the similarity among spectra. Prediction maps are PLS-DA results: each class is defined by a different colour.

\section{Results}

The spectra of the different materials, collected in the NIR range, provides chemical information about their composition, being spectral signatures absorption bands strictly related to $\mathrm{C}-\mathrm{H}, \mathrm{O}-\mathrm{H}$ and $\mathrm{N}-\mathrm{H}$ overtones.

\subsection{Explorative analysis and class setting}

The explorative analysis was carried out after spectra preprocessing applying Mean Center, Detrend and SNV algorithms.

The sample set corresponding to the Experimental set up 1 was analyzed by PCA: the majority of the variance was captured by the first two PCs, where PC1 and PC2 explained $58.43 \%$ and $25.03 \%$ of the variance, respectively.

The six classes were clearly identified on the PCA score plot because samples, in fact, are clustered into six different groups (Figure 12.3). Therefore the training dataset was easily created by removing some border cluster points "different" from all other spectra of the same category and by setting class of the remaining pixels. 


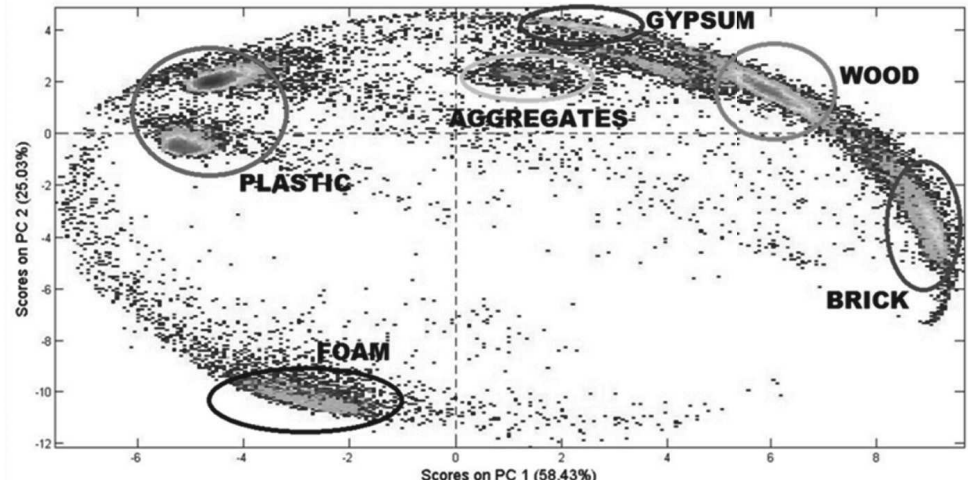

Figure 12.3: PCA score plot after preprocessing: all the classes are identified.

\subsection{PLS-DA classification}

In order to classify the sample set corresponding to the Experimental set up 2, a PLS-DA method was applied.

The number of latent variables was chosen based on the minimum classification error in the venetian blind cross-validation and it was 5 LVs and the obtained values of Sensitivity and Specificity are shown in the Table 12.1. These parameters are the model efficiency indicators: the more the values are close to one, the better the modelling.

Table 12.1: Sensitivity and Specificity for the PLS-DA model built for the classification purpose.

\begin{tabular}{cccccc}
\hline $\begin{array}{c}\text { Applied algorithms } \\
\text { in } \\
\text { the PLS-DA model }\end{array}$ & Class & \multicolumn{2}{c}{$\begin{array}{c}\text { Sensitivity } \\
\text { Calibration }\end{array}$} & $\begin{array}{c}\text { Specificity } \\
\text { Calidation }\end{array}$ & \multicolumn{2}{c}{ Calibration } & $\begin{array}{c}\text { Cross } \\
\text { Validation }\end{array}$ \\
\hline & Aggregates & 0.997 & 0.997 & 0.002 & 0.002 \\
& Brick & 0.988 & 0.988 & 0.996 & 0.996 \\
& Gypsum & 0.982 & 0.981 & 0.999 & 0.999 \\
Detrend, SNV, Mean Center & Plastic & 1.000 & 1.000 & 1.000 & 1.000 \\
& Wood & 0.985 & 0.985 & 0.939 & 0.939 \\
& Foam & 1.000 & 1.000 & 1.000 & 1.000 \\
\hline
\end{tabular}


The Sensitivity, true positive rate, estimates the model ability to avoid false negatives (i.e. number of samples of a given type correctly classified as that type). The Specificity is the estimation of the model ability to avoid false positives (the number of samples not of a given type correctly classified as not of that type).

Sensitivity and Specificity are very close to 1, except for the Specificity of aggregates: there are many false positive, probably due to dirtiness on the particles surface. Indeed, these materials were not washed, so some pixels, corresponding to pollutant, can have a similar spectra of aggregates distorting the classification result. Furthermore there are some light scattering problems due to the "rough" and heterogeneous particles surface that can contribute to the presence of errors and/or misclassifications.

In order to verify its classification ability, the built model was applied to the image corresponding to the Experimental set up 2. The results, in form of prediction maps, are reported in Figure 12.4a. The built model gives a good classification even if some sporadic misclassifications occur, but it is easy to indentify the predominance of a single class on each object. Therefore it is possible to impose a constraint on the maximum percentage of pixels assigned to each object in the image: the class with the major number of pixels on an object is assigned to the entire object, to reach this goal the percentage of pixels belonging to each class for every object in the image was measured.

In Figure $12.4 \mathrm{~b}$ are reported the labels for the particles of the Experimental set up 2 used to build the Table 12.2, showing the percentages of pixels belonging to each class for every particle.

A new classification was obtained after the imposition of the constraint rules about the maximum percentage of assigned pixels to a specific class (Figure 12.5).

\section{Conclusions}

The possibility to develop an efficient recovery and reuse of concrete materials, considered as secondary raw materials, represents one of the main targets in the DW sector. The achievement of this goal can contribute to decrease the steady supplies of good quality natural aggregates but in the same time it can secure large supplies of concrete ag- 


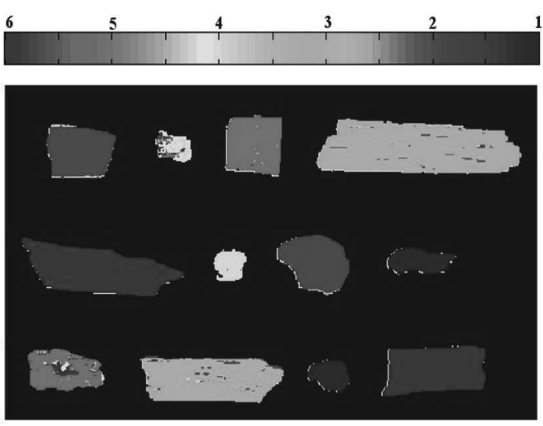

a

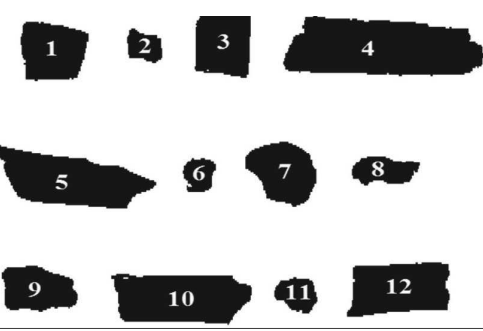

b

Figure 12.4: Experimental set up 2: (a) prediction maps obtained as result of PLS-DA classification model where classes are: foam (1), gypsum (2), wood (3), aggregates (4), brick (5) and plastic (6); (b) labels corresponding to each classified particles.

Table 12.2: Percentage of pixels belonging to each class (i.e. foam, gypsum, wood, aggregates, brick and plastic) in every particle of the Experimental set up 2 .

\begin{tabular}{lcccccccccccc}
\hline & \multicolumn{10}{c}{ Particles } \\
\hline Materials (\%) & 1 & 2 & 3 & 4 & 5 & 6 & 7 & 8 & 9 & 10 & 11 & 12 \\
\hline Foam & 0 & 0 & 0 & 0 & 0 & 0 & 0 & 100 & 0 & 0 & 100 & 0 \\
Gypsum & 97.31 & 0.74 & 0 & 2.82 & 0 & 0.25 & 98.35 & 0 & 0.16 & 6.63 & 0 & 0 \\
Wood & 0.85 & 0.25 & 0 & 95.7 & 0 & 0.5 & 1.65 & 0 & 0 & 90.23 & 0 & 0 \\
Aggregates & 1.84 & 71.51 & 0.53 & 1.51 & 0 & 98.75 & 0 & 0 & 4.76 & 3.02 & 0 & 0 \\
Brick & 0 & 14.1 & 99.3 & 0 & 0 & 0 & 0 & 0 & 82.71 & 0.12 & 0 & 0 \\
Plastic & 0 & 13.4 & 0.13 & 0 & 100 & 0.5 & 0 & 0 & 12.37 & 0 & 0 & 100 \\
\hline
\end{tabular}

gregates to the construction industry and moreover it can reduce the environmental constraints linked to the DW wastes disposal, especially in urban regions. Several benefits can be thus reached: i) the reduction of new non-renewable resources exploitation and of the costs linked to transport and energy production, ii) the possibility to utilize materials that otherwise should be lost (i.e. land filled), iii) the land preservation in respect of future urban development and, finally, iv) the reduction of the impact, on the environment, of new exploitation activities of 


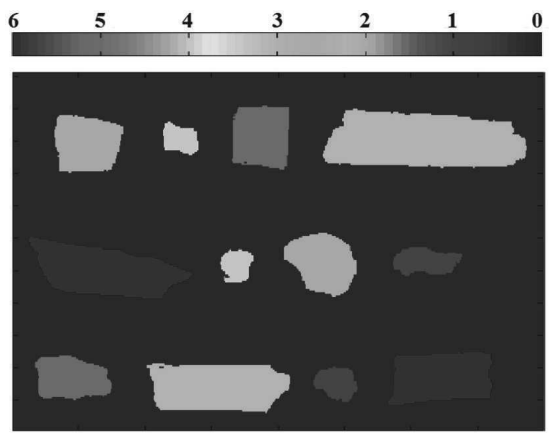

Figure 12.5: New classification, as resulting from Experimental set up 2, after the application of the constraint rules about the maximum percentage of assigned pixels to each class: background (0); foam (1); gypsum (2); wood (3); aggregates (4); brick (5); plastic (6).

non-renewable resources. Starting from these premises the possibility to identify a suitable sensor technology for quality measurement and control of recycled streams along the entire chain, from demolition to "new" concrete and/or mortar production, able to recognize and characterize recycled aggregates, as well as the presence of contaminants can represent an important starting point to develop, implement and set up on-line control strategies to apply before and after demolition actions. In this study a procedure based on HyperSpectral Imaging in the NIR region was thus developed in order to identify different materials in a DW stream: five kind of contaminants (i.e. brick, plastic, gypsum, wood and foam) and recycled aggregates. Analysis were carried out adopting chemometric methods: PCA for the exploratory analysis and PLS-DA for the classification purpose. Obtained results showed that the classification was good and sporadic misclassifications are probably due to light scattering, samples heterogeneity and presence of impurities. In order to improve the classification, a logic defining the maximum percentage of assigned pixels to each class for every particle was implemented: only one class was thus attributed to each object in the image to be predicted. The proposed HSI based approach is not-expensive, fast and reliable. For all these reasons it can be profitably utilized to set up on-line strategies finalized to improve recycling processes efficiency, 
reducing costs and improving the "final quality" of the recovered products.

\section{Acknowledgments}

This study is funded by the European Commission in the framework of the FP7 Collaborative project "Advanced Technologies for the Production of Cement and Clean Aggregates from Construction and Demolition Waste (C2CA)". Grant Agreement No 265189.

\section{References}

1. F. Pacheco-Torgal, "Introduction of construction and demolition waste (cdw)," in Handbook of recycled concrete and demolition waste. Woodhead Publishing Limited, Cambridge, 2013.

2. F. Martín-Morales, M. Zamorano, I. Valverde-Palacios, G. Cuenca-Moyano, and Z. Sánchez-Roldán, "Quality control of recycled aggregates (ras) from construction and demolition waste (cdw)," in Handbook of recycled concrete and demolition waste. Woodhead Publishing Limited, Cambridge, 2013.

3. T. Hyvarinen, E. Herrala, and A. Dall'Ava, "Direct sight imaging spectrograph: a unique add-on component brings spectral imaging to industrial applications," in Proc. SPIE Electronic Imaging, vol 3302, San Jose, CA, USA, January 1998.

4. P. Geladi, H. Grahn, and J. Burger, "Multivariate images, hyperspectral imaging: background and equipment," in Techniques and Applications of $\mathrm{Hy}$ perspectral Image Analysis. Jhon Wiley \& Sons, West Sussex, England, 2007.

5. P. Geladi, J. Burger, and T. Lestander, "Hyperspectral imaging: calibration problems and solutions," in Chemometrics and Intelligent Laboratory Systems, vol.72, 2004.

6. W. de Vries, P. Rem, and S. Berkhout, "Adr: a new method for dry classification," in Proc. of the ISWA International Conference, Lisbon, Portugal, 2009.

7. R. Palmieri, G. Bonifazi, and S. Serranti, "Automatic detection and classification of eol-concrete and resulting recovered products by hyperspectral imaging," in Proc. SPIE Advanced Environmental, Chemical, and Biological Sensing Technologies XI, vol 9106, Baltimore, Maryland, USA, 2013.

8. B. Wise, N. Gallagher, R. Bro, J. Shavar, W. Winding, and R. Koch, “Pls toolbox user manual." Eigenvector Research Inc., 2005. 
9. S. Wold, K. Esbensen, and P. Geladi, "Principal component analysis," in Chemometrics and Intelligent Laboratory Systems, vol.2, 1987.

10. J. Amigo, I. Marti, and A. Gowen, “Hyperspectral imaging and chemometrics: a perfect combination for the analysis of food structure, composition and quality," in Data Handling in Science and Technology, Vol.28. Federico Marini (Ed.), Amsterdam: The Netherlands, 2013.

11. M. Barker and W. Rayens, "Partial least squares for discrimination," in Journal of Chemometrics, Vol.17, 2003.

12. D. Ballabio and V. Consonni, "Classification tools in chemistry. part 1: linear models. pls-da," in Analytical Methods, Vol.5, 2003. 
